\title{
OPORTUNIDADES DE INTEGRACIÓN PARA LOS LLEGADOS EN LA INFANCIA. CAMBIOS EN EL PERFIL TRADICIONAL DE LOS MEXICANOS EN ESTADOS UNIDOS
}

\author{
Integration opportunities for childhood arrivals. \\ Changes in the traditional profile of Mexicans in the United States
}

\author{
Selene Gaspar Olvera* \\ Rodolfo García Zamora**
}

\begin{abstract}
Resumen. La emigración de mexicanos ha sido predominantemente en edades laborales y reproductivas, a ese contingente se han sumado a lo largo del tiempo los llamados "llegados en la infancia" que se caracterizan por haber ingresado a Estados Unidos cuando eran menores de edad. Algunos se han establecido de manera permanente a través de un medio legal; otros, en cambio, permanecen con estatus irregular de residencia, situación que supone oportunidades diferenciadas en su desarrollo personal y profesional, y de integración a la sociedad estadounidense. Observamos que aun entre los no naturalizados la edad de llegada temprana favorece cambios en el capital humano de los inmigrantes mexicanos y diversifica su participación ocupacional, maximizando las posibilidades de integración y su aporte a la sociedad de residencia. La ciudadanía estadounidense obtenida por naturalización es un mejor predictor de integración pues les permite acceder a derechos políticos y sociales y reduce la desigualdad salarial.
\end{abstract}

Palabras clave: llegados en la infancia; soñadores; mexicanos; Estados Unidos; integración.

\begin{abstract}
The emigration of Mexicans has been predominantly in working and reproductive ages, to this contingent have been added over time the so-called "childhood arrivals" characterized by having entered the United States when they were minors. Some have been established permanently through legal means; others, on the other hand, remain with irregular residence status, a situation that implies different opportunities in their personal and professional development, and integration into American society. We observe that even among the non-naturalized, the early arrival age favors changes in the human capital of Mexican immigrants and diversifies their occupational participation, maximizing the possibilities of integration and their contribution to the society of residence. US citizenship obtained by naturalization is a better predictor of
\end{abstract}

\footnotetext{
Universidad Autónoma de Zacatecas. Zacatecas, México. E-mail: selene.gasparolvera@gmail. com. Orcid: https://orcid.org/0000-0002-1312-5385.

** Universidad Autónoma Zacatecas. Unidad Académica de Estudios del Desarrollo. Zacatecas, México. E-mail: rgarciazamora54@gmail.com. Orcid: https://orcid.org/0000-0002-0685-3165.
} 
integration as it allows them access to political and social rights and reduces wage inequality.

Keywords: childhood arrivals; dreamers; Mexicans; United States; integration.

\section{Introducción ${ }^{1}$}

Estados Unidos durante mucho tiempo ha sido considerado por millones de migrantes un lugar donde se puede prosperar cuando se trabaja duro, un lugar donde tener un trabajo seguro, formar un hogar y forjar un futuro para sus hijos. Hay evidencia de que los inmigrantes, documentados e indocumentados, de todos los tiempos y niveles de calificación, durante mucho tiempo han contribuido al crecimiento y dinamismo de la economía de una de las naciones más prosperas del mundo. Para el gobierno de Estados Unidos, hoy en día como en el pasado la cuestión de la inmigración en situación irregular de residencia, también conocidos como indocumentados, es un tema sensible y polarizado. Para Lee Youngro (2006) las posibles soluciones a la inmigración indocumentada están destinadas a ser complejas e inciertas en virtud de la gran cantidad de cuestiones inherentemente asociadas a ella.

Un grupo central de la inmigración indocumentada son los llegados en la infancia, conformado por inmigrantes que fueron llevados a Estados Unidos por sus padres o familiares cuando eran niños de manera indocumentada; algunos con el tiempo regularon su situación de residencia a través de la naturalización y con permisos de residencia temporal; otros, en cambio, han permanecido en condición indocumentada, situación que supone oportunidades diferenciadas en su desarrollo personal y profesional, y de integración a la sociedad estadounidense (Gaspar, 2018).

Para los llegados en la infancia que han permanecido en Estados Unidos de manera indocumentada se han introducido una serie de propuestas para regular su situación de residencia permanente, sin que ninguna de ellas haya tenido éxito. En 2001 se introduce un proyecto de ley bipartidista en el senado, la Ley de Desarrollo, Ayuda y Educación para Menores Extranjeros (DREAM-S.1291), cuyo objetivo era proporcionar un medio para obtener estatus legal permanente para los inmigrantes indocumentados que llegaron a Estados Unidos cuando eran niños. Desde entonces se han introducido numerosas versiones del DREAM (LawLogix, 2013; American Immigration Council, 2017).

Una de las propuestas que se ha mantenido a pesar de los continuos ataques que ha tenido por parte del mandatario Donald Trump es el programa DACA (Acción Diferida para los Llegados en la Infancia), programa que se aprobó el 15 de junio de 2012, durante el periodo del gobierno Barack Obama. Cabe señalar

\footnotetext{
1 Annual Meeting of the Population Association of America, April 10 to April 13, 2019, in Austin, Texas. Wellbeing of Mexican-Origin Children on Both Sides of the U.S./Mexico Border. Revisión Dra. Jenna E Nobles.
} 
que el programa no ofrece un camino hacia la residencia permanente legal (LPR) o la ciudadanía estadounidense, es un permiso temporal de residencia o trabajo. A marzo de 2020 se aceptaron 2,798,910 solicitudes de las cuales 2,646,963 (94.6\%) se aprobaron (94.6\%), de estas últimas 2,091,214 (79.0\%) se otorgaron a los Ilegados en la infancia procedentes de México, 68.9\% de los cuales fueron renovaciones (USCIS, 2020). Las cifras en sí misma muestran la importancia que tiene mantener DACA o abrir opciones que les permita regular su situación migratoria para los llegados en la infancia.

Para los llegados en la infancia en situación irregular de residencia no ha sido fácil vivir escondiéndose y superando las restricciones de acceso a la educación, a la salud y al bienestar; aun así hay numerosos ejemplos de que estas personas mejoran su capital humano y situación económica cuando se abren oportunidades de desarrollo para ellos, tales como, asistir a la escuela, acceso a la salud y al mercado laboral. Alejandro Portes y Rubén Rumbaut (2010) señalan que para los migrantes el contexto social juega un papel decisivo en sus perspectivas económicas y de desarrollo. Para los llegados en la infancia es decisivo el contexto social pues de ello depende el capital humano que logran alcanzar, que a su vez será determinante en su movilidad social, profesional, laboral y económica. Para Santibáñez y Lavore (2012) los jóvenes migrantes son más susceptibles de asentarse definitivamente en el país de destino ya que aún no desarrollan lazos familiares fuertes ni redes sociales en su país de origen. Además de que pueden hacerse con mayor facilidad de recursos para insertarse en la sociedad de destino como son el aprendizaje del idioma inglés y el acceso a los servicios de salud y a la educación. Acceso que estará determinado por su estatus legal de residencia, así como de la movilidad social y económica de sus padres.

La gran mayoría de los inmigrantes mexicanos se ha caracterizado por su perfil de baja calificación y ha emigrado a Estados Unidos en edad laboral; en cambio, los llegados en la infancia han alcanzado cierto nivel de capital humano en las escuelas de Estados Unidos y crecido y socializado en sus comunidades de asentamiento en ese país. Con el objetivo de aportar elementos que evidencien cambios en el patrón tradicional de baja escolaridad observados en los inmigrantes que llegan a una edad adulta respecto de los que llegaron en la infancia analizamos las características sociodemográficas y laborales de los inmigrantes mexicanos por edad de llegada a Estados Unidos y estatus de ciudadanía. Recurrimos al estatus de ciudadanía como un proxi al estatus de residencia legal, toda vez que la encuesta que utilizamos para el estudio no distingue entre documentados e indocumentados. Interesa mostrar que los inmigrantes mexicanos que llegaron en la infancia que no se han naturalizado o regulado su situación migratoria mejoran su perfil educativo y laboral respecto de los que llegan a una edad adulta, lo cual favorecería abrir oportunidades de integración para ellos.

Es posible que los inmigrantes mexicanos que llegaron a una edad temprana y han tenido la oportunidad de naturalizarse, completen más años de escolaridad, 
tengan mayor dominio del idioma inglés y logren mejores condiciones en el mercado laboral que aquellos que no se han naturalizado o regulado su situación de residencia legal, pero estos último tendrán un perfil promedio socioeconómico y laboral más similar que los que llegaron a una edad adulta. Los inmigrantes se integran cuando hay oportunidades de desarrollo y cohesión, es decir, cuando el país de destino los integra a la sociedad, lo cual con frecuencia ocurre cuando el migrante ajusta su estatus de residencia permanente legal (LPR) o adquieren la ciudadanía a través de la naturalización (Waters, Gerstein, 2015).

Encontramos para los mexicanos que llegaron en la infancia a Estados Unidos, que si bien, la obtención de la ciudadanía favorece cambios en su capital humano, lo cual diversifica su participación ocupacional y mejora sus salarios, maximizando así las posibilidades de integración para ellos y aportes económicos para el país en el que crecieron, la edad temprana de llegada a ese país para los que no se han naturalizado también es un factor que favorece perfiles más calificados para su integración.

\section{Métodos y datos}

El estudio se lleva a cabo con estadística derivada de la American Community Survey (ACS) 2017. La ACS es una encuesta de hogares que se levanta mensualmente desde el año 2000, su diseño permite contar con micro datos anuales, trienales o quinquenales. El tamaño aproximado de la ACS anual, a partir de 2005, es de 3 a 3.5 millones de direcciones al año y a partir del levantamiento de 2006 incluye a todos los residentes actuales no institucionales (viviendas particulares) y en instituciones y cuarteles militares (viviendas colectivas), recopila información detallada sobre las viviendas y características demográficas, sociales, económicas y laborales de la población en su residencia actual en forma permanente durante los 12 meses del año.

La ACS no distingue el estatus migratorio del inmigrante, es decir, su condición de residencia legal, por lo que la variable de ciudadanía que identifica a los inmigrantes que se han naturalizado y por lo tanto regulado su situación de residencia legal es una manera aproximada de acércanos al estatus legal de residencia del inmigrante. No obstante, se tiene presente que esta aproximación puede estar sobreestimada pues hay otros mecanismo legales para residir de manera legal y permanente en Estados Unidos, como son patrocinio de un familiar o empleador, mediante estatus de refugiado o asilado, por mencionar algunas formas (véase American Inmigración Council, 2019).

En un primer apartado se analizan las condiciones actuales de integración social, económicas y laborales de los inmigrantes mexicanos que llegaron con sus padres o un familiar antes de su $16^{\circ}$ aniversario a Estados Unidos y tienen entre 21-70 años de edad, se compara aquellos que han obtenido la ciudadanía estadounidense respecto de los que no lo han hecho. Para simplificar el análisis 
a este grupo lo denominamos "Llegados en la infancia" y los clasificamos en dos grupos (1) Llegados en la infancia con ciudadanía estadounidense y (2) Llegados en la infancia sin ciudadanía estadounidense. La edad de 21 años corresponde a la edad en que una persona se convierte en adulto en Estados Unidos y la edad de 70 años corresponde a la edad de jubilación, este rango de edades comprende a la población en edad de mayor producción laboral y nos permite observar grupos comparables. Utilizamos un segundo grupo de contraste, el de los inmigrantes mexicanos que llegaron a Estados Unidos con 16 años o más de edad y tienen entre 21-70 años de edad con cinco años o más de residencia en Estados Unidos, grupo que denominamos como "Llegados con 16 años o más", los cuales son clasificados por condición de ciudadanía, (3) Llegados con 16 años o más con ciudadanía estadounidense y (4) Llegados con 16 años o más sin ciudadanía estadounidense.

Se utilizan técnicas de estadística descriptiva y mediante la aplicación del Índice de Disimilitud de Duncan y Duncan (1955) se estima la distancia o diferencia que guardan los grupos en análisis unos de otros, respecto a sus indicadores sociales, económicos y laborales. El Índice de Duncan y Duncan (ID) es igual a la suma de la proporción del grupo xi que debe cambiar para alcanzar la igualdad en el indicador i. Entre las principales bondades del ID se encuentran que su procedimiento de cálculo es sencillo de aplicar y permite adecuar su uso o aplicación para distintas disciplinas. El índice corre sobre un rango [0, 1], la interpretación del índice es: valores cercanos a 0 indican mínima desigualdad mientras que cercanos a 1 indican máxima desigualdad. Es decir, 0 corresponde a una distribución exactamente igualitaria y 1 a una distribución de máxima desigualdad. Para simplificar su interpretación se multiplica por 100 (véase Gaspar, 2018). Este procedimiento requiere un grupo de referencia, que será el que marque las distancias entre los grupos. El grupo de referencia son los inmigrantes mexicanos que llegaron en la infancia con ciudadanía estadounidense (grupo 1). La información que se presenta en el Cuadro 1 agrupa los indicadores analizados en indicadores de capital humano, culturales e indicadores laborales. Los términos ciudadanía estadounidense y naturalizada se usan de manera indistinta.

Procedimiento del índice tomado de Gaspar (2018).

Sea $M_{i}=\frac{m_{i}}{M}$ la proporción del grupo $\mathrm{j}=1,2,3$ en el indicador $\mathrm{i}$ Sea $N_{i}=\frac{n_{i}}{N}$ la proporción de nativos blancos no hispanos en el indicador i

$$
I D=\frac{1}{2} * \sum\left|N_{i}-M_{i}\right|
$$

La cifra $1 / 2$ se necesita para normalizar el índice y corre sobre un rango [0, 1].

En este trabajo se entiende por "integración el proceso social mediante el cual inmigrantes y nativos se involucran en los diversos ámbitos de la sociedad de residencia (familiar, comunitaria, económica, social, política, territorial y cultural), que depende de las políticas y prácticas de la comunidad de asentamiento $y$, por lo tanto, de las herramientas, acciones y oportunidades que permiten su 
autonomía individual y su participación en la sociedad" (Gaspar, 2018, p. 180). Dado que nuestro análisis utiliza datos de una encuesta transversal, no estudiamos la asimilación y por ello no recurrimos a esa teoría.

Cuadro 1 - Indicadores de capital humano, sociales, culturales y económicos; y variables para el análisis laboral

\begin{tabular}{|c|c|c|}
\hline Indicadores de capital humano & Indicador cultural & Indicadores laborales \\
\hline $\begin{array}{l}\text { 1) \% de población que habla muy } \\
\text { bien y bien el idioma inglés } \\
\text { 2) \% de población que tiene } \\
\text { estudios superiores (incluye: grado } \\
\text { asociado a licenciatura, licenciatura } \\
\text { o posgrado) }\end{array}$ & $\begin{array}{l}\text { 1) \% en hogares lingüísticamente } \\
\text { no aislados: hogares donde todos } \\
\text { sus miembros mayores de } 14 \text { años } \\
\text { hablan solo inglés } / 1 \text {. }\end{array}$ & $\begin{array}{l}\text { 1) Condición de actividad } \\
\text { 2) Tipo de ocupación } \\
\text { 3) Tipo de empleado } \\
\text { 4) Salario por trabajo }\end{array}$ \\
\hline Indicadores sociales & Indicadores económicos & \\
\hline $\begin{array}{l}\text { 1) \% población que asiste a la } \\
\text { escuela ( } 6 \text { a } 21 \text { años de edad) }\end{array}$ & $\begin{array}{l}\text { 1) \% con vivienda propia o a crédito } \\
\text { 2) \% con vehículo en la vivienda }\end{array}$ & \\
\hline
\end{tabular}

Nota: 1/ El entorno del lenguaje en el hogar está asociado con diferencias en el desarrollo temprano y puede servir como una barrera para la integración y acceso a servicios. Los inmigrantes que tienen más movilidad ascendente también pueden ser más propensos a adquirir inglés y salir de comunidades lingüísticamente aisladas (E. Glick, Walker y Luz (2013).

Fuente: Elaborado con base en Integración de los inmigrantes mexicanos que llegaron en la infancia a Estados Unido, CONAPO. Situación Demografía de México, 2017.

\section{La edad de llegada, la ciudadanía y los llegados en la infancia}

La edad a la que se inicia el proceso migratorio y se llega al nuevo país de residencia define etapas del ciclo de vida de estas personas, tales como incorporarse o reincorporarse al sistema escolar, la entrada al mercado laboral, casarse, tener hijos, etcétera; y es un determinante clave de su adaptación e integración a la sociedad de acogida (Rumbaut, 2006). Los inmigrantes que llegaron en la infancia a Estados Unidos que han permanecido en condición indocumentada son un grupo altamente vulnerable a la deportación y desintegración familiar, ante el cierre de apertura de canales legales para regular su situación migratoria y la postura hacia la inmigración del actual gobierno. Sin embargo, la postura de Trump no es respaldada, el $74 \%$ de los estadounidenses está a favor de una ley que otorgue un estatus legal permanente para los inmigrantes que llegaron a Estados unidos ilegalmente cuando eran niños, casi en proporciones similares (54\%) entre demócratas y republicanos opinan lo mismo, sin embargo es más probable que los demócratas expresen su apoyo (89\% frente a 57\%) (Krogstad, 2020).

Para quienes participan en el proceso migratorio, la edad en que se emprende el viaje o se llega al país de destino configura las capacidades sociales, emocionales y cognitivas de una manera tal que afecta los resultados en la edad adulta (Hermansen, 2013). Los menores inmigrantes que crecen en entornos desfavorecidos experimentan mayores dificultades para lograr un pleno desarrollo, acumulan desventajas y resultados socioeconómicos adversos; sin embargo, 
la evidencia empírica muestra que cuando más niño se llega al país de destino las probabilidades de adaptación, aprendizaje del idioma del país anfitrión y socialización se incrementan (Rumbaut, 2006). Se ha encontrado que los niños migrantes aprenden un nuevo idioma más fácilmente que los llegan a edades mayores, así como el efecto positivo que tiene la competencia lingüística del país anfitrión en los salarios de los inmigrantes (Bleakley, Chin, 2004). De acuerdo con Baum y Flores (2011), los niños que emigran antes de los 12 años están más expuestos al lenguaje, a las normas del sistema educativo y son mejores en la escuela que aquellos que emigran a edades posteriores.

Sobre el estatus migratorio Santibáñez y Lavore (2012), resaltan como una cuestión central en materia de migratoria para los países receptores de migrantes, la necesidad de abrir canales legales para que los inmigrantes se conviertan en ciudadanos, a través de la naturalización, en las sociedades a donde llegan. Pues la ciudadanía permite a los inmigrantes acceder a derechos políticos y sociales, por lo tanto, facilita la integración en las sociedades de llegada. Los inmigrantes mexicanos en Estados Unidos tienen un elevado índice de indocumentación y bajos niveles de ciudadanía, para tales autores esto constituye un obstáculo decisivo para su integración a la sociedad estadounidense y, por lo tanto, restringe el acceso a beneficios económicos, sociales y de desarrollo. La falta de documentos legales de residencia es un tema transversal en la vida de los inmigrantes, pues limita severamente sus posibilidades laborales y educativas en Estados Unidos. Peor aún, la falta de documentos legales de residencia violenta los derechos humanos de los inmigrantes, en tanto que vulnera su acceso a la justicia, e incluso a experimentar discriminación por parte de autoridades, situación que los empuja a mantenerse alejados de las instancias judiciales cuando sufren agresiones, amenazas o alguna otra forma de abuso, además del riesgo latente de ser deportado en cualquier momento.

Borjas y Tienda (1993) encontraron que los inmigrantes legalizados durante el IRCA ganaban 30 por ciento más que los no legalizados. Por su parte, Pastor y Scoggins (2012) señalan que incluso cuando se controla por una serie de factores como el nivel de escolaridad y el origen nacional, los inmigrantes naturalizados ganan once por ciento más que los que no se han naturalizado. Enchautegui y Giannarelli (2015) estiman que México es el principal país de origen de la población elegible para la naturalización en 9 de 21 ciudades.

La literatura sobre la edad de llegada de los inmigrantes y el tiempo de estancia en Estados Unidos, permiten suponer una asociación positiva con la obtención de la residencia permanente legal (LPR) o para adquirir la ciudadanía a través de la naturalización. Lo anterior es así, cuando hay una apertura para el desarrollo y la cohesión; pues el proceso de integración, tal como lo señalan G. Rumbaut y Ewing (2007) a menudo implica el dominio del idioma inglés y niveles más altos de educación para adquirir habilidades y atributos laborales valiosos para la economía del país, así como, para la adaptación a la vida y cultura de la sociedad estadounidense. Atributos que se encuentran dentro de los requisitos para obtener LPR o la ciudadanía. 
La situación irregular de residencia con el que fueron traídos los Dreamers o llegados en la infancia a Estados Unidos y la política de inmigración implementada desde el IRCA ha creado un contexto adverso que los excluye y limita sus posibilidades de desarrollo y de beneficios básicos de bienestar. A los inmigrantes en situación irregular de residencia que concluyen sus estudios de postsecundaria disfrutar de la movilidad ascendente que la educación superior permite, al prohibir beneficios de educación superior a nivel estatal (Yates, 2004).

En cualquier escenario que se plantee, y sin temor a equivocarse, negar el acceso a la educación solo incurrirá en altos costos para el Estado y contribuirá a una fuerza laboral de baja calificación que redundará en bajas recaudaciones fiscales. "La educación en el marco de la igualdad,..., y es inestimable los costos de esa privación en lo social, económico, bienestar intelectual y psicológico del individuo" (Plyler, 1982, 457 U.S. at 221, citado en Yates, 2004, p. 591). A este tenor cualquier intento de segregación por raza u origen étnico solo fortalece el racismo y dificulta la integración social, económica, laboral, residencial y cultural de los inmigrantes.

Para Water y Gerstein (2015) a menudo las personas transitan entre diferentes estados de inmigración, estas fases determinan diferencias en el derecho a permanecer en Estados Unidos, en los derechos a beneficios públicos, a los servicios del gobierno y en la capacidad de trabajar y participar plenamente de la vida social y cívica de la nación de acogida. Así mismo, señalan que los inmigrantes realmente comienzan el proceso de integración a la sociedad estadounidense cuando ajustan su estatus de residencia permanente legal.

Woodrow, Xu, Kersen y Poch (2004) señala que los inmigrantes tienen diferentes motivaciones para emigrar y poseen diferente capital humano o potencial para su integración a la sociedad de destino. Sin embargo, los inmigrantes que llegaron en la infancia a Estados Unidos generan su capital humano y potencial no solo para su integración social y económica, también, para la aculturación, lo cual depende de múltiples factores incluidos su estatus de residencia, oportunidades de desarrollo y de cohesión que están disponibles para ellos. De ahí la importancia del contexto social mencionado por Portes y Rumbaut pues el nivel de capital humano y aculturación que alcanzan los inmigrantes que llegaron en la infancia repercute en sus posibilidades de legalizarse o naturalizarse, en tanto que, en ambos casos, de ello depende el cumplimiento de los requisitos establecidos para tal fin.

\section{Dimensión de los llegados en la infancia}

Previo al análisis de nuestra poblaciones objetivo definidas en el apartado de métodos y datos, dimensionamos el número de inmigrantes que llegaron en la infancia antes de su 16 aniversario a Estados Unidos. Los llegados en la infancia en Estados Unidos construyeron una vida en el país en el que crecieron, estudian o estudiaron en sus escuelas y/o trabajan en el mercado laboral del país, así 
contribuyen al crecimiento económico de la nación a la llegaron siendo niños y que ha sido su hogar durante sus vidas. En 2017, más de 13.6 millones (28.6\%) de los 47.6 millones de inmigrantes en Estados Unidos llegaron a ase país antes de su 16음 aniversario, uno de cada cuatro es un inmigrante mexicano. De los 13.6 millones, 11.8 millones (86.8\%) tiene por lo menos cinco años o más de estancia en ese país.

Entre los Ilegados en la infancia que no se han naturalizado, que incluye al grupo de inmigrantes en situación irregular de residencia, suman en 2017, 3.1 millones (28.7\%), este grupo tienen una media de 23 años de residencia, una edad promedio de 32 años y llegaron cuando tenían en promedio 8 años de edad, personas cuyas vidas se han reducido a un mero número y ahora son objeto de negociación (Eng, 2018).

Entre los inmigrantes indocumentados, el de mayor controversia por su número ha sido la de los mexicanos, estimamos que 3.5 millones de los inmigrantes mexicanos ingresó a Estados Unidos antes de cumplir su 16ㅇaa aniversario. Tres millones (86.6\%) de los cuales en 2017 tenía 16 años o más de edad. De los 3 millones, 43.5\% (1.3 millones) se ha naturalizado, los restantes 1.7 millones tiene residencia legal o son indocumentados. Los inmigrantes mexicanos encabezan el grupo de inmigrantes que llegaron en la infancia, ya sea entre los que tienen menos de 16 años de edad o entre los que tienen 16 años o más de edad, 32.4\% y 54.9\% respectivamente. Entre los menores no naturalizado o con estatus irregular de residencia sobresalen por su número los procedentes de la India (8.2\%), Republica Dominicana (4.3\%), Filipinas (3.1\%) y el Salvador $3.0 \%$ ) en las primeras cinco posiciones. Entre los mayores, es decir, los que tienen 16 años o más de edad, México agrupa al 54.9\%, le siguen El Salvador con 4.3\%, Guatemala con 3.0\% y Canadá con el 2.3\% (Cuadro 2).

Cuadro 2 - Principales países de origen de los inmigrantes que llegaron antes de cumplir su 160 aniversario y tienen cinco años o más de residencia en Estados Unidos, por condición de ciudadanía, 2017

\begin{tabular}{|c|c|c|c|c|c|}
\hline \multicolumn{3}{|c|}{$\begin{array}{c}\text { Menores de } 16 \text { años de edad con } 5 \text { años o más } \\
\text { de residencia }\end{array}$} & \multicolumn{3}{|c|}{$\begin{array}{l}\text { Mayores de } 16 \text { años de edad con } 5 \text { años o más } \\
\text { de residencia }\end{array}$} \\
\hline Total & 998,523 & 100.0 & & $10,808,460$ & 100.0 \\
\hline \% con ciudadanía & 599,463 & 60.0 & & $7,704,917$ & 71.3 \\
\hline \multicolumn{6}{|c|}{ Sin Ciudadanía estadounidense o estatus irregular de residencia } \\
\hline Total & 399,060 & 100.0 & & $3,103,543$ & 100.0 \\
\hline México & 129,210 & 32.4 & México & $1,704,849$ & 54.9 \\
\hline India & 32,718 & 8.2 & El Salvador & 134,216 & 4.3 \\
\hline República Dominicana & 17,194 & 4.3 & Guatemala & 94,516 & 3.0 \\
\hline Filipinas & 12,383 & 3.1 & Canadá & 71,422 & 2.3 \\
\hline El Salvador & 11,971 & 3.0 & República Dominicana & 67,060 & 2.2 \\
\hline
\end{tabular}

Fuente: Estimación propia con base en U.S. Census Bureau, American Community Survey (ACS), 2017. 
Como se puede observar en la información del Cuadro 2 los llegados en la infancia que podrían participar en el programa DACA rebasan los 3 millones. El número de beneficiarios del programa DACA se ha visto limitado entre otros factores por lo requisitos de inscripción que limita la edad a menos de 31 años al 15 junio de 2012 (USCIS). Entre los llegados en la infancia procedentes de México sin ciudadanía estadounidense (1.8 millones) casi cuatro de cada diez queda fuera de toda posibilidad para incorporase al programa por el requisito de la edad, pues 39.4\% tiene entre 31 años o más (Gráfica 1).

Gráfica 1 - Inmigrantes mexicanos que llegaron a Estados Unidos antes de cumplir su 160 aniversario y tiene cinco años o más de residencia, 2017

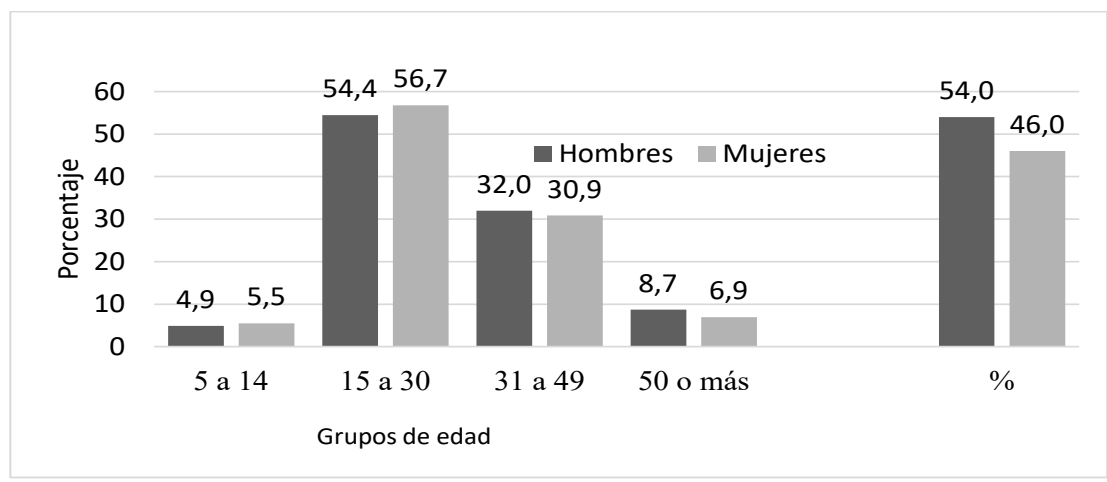

Fuente: Estimación propia con base en U.S. Census Bureau, American Community Survey ACS 2017.

\section{Oportunidades de integración. Logros de los adultos que llegaron en la infancia}

A partir de aquí, retomamos a la población objetivo definida en el apartado de métodos y datos, nos centramos en los inmigrantes mexicanos que ingresaron a Estados Unidos antes de cumplir su $16^{\circ}$ aniversario y tienen edades comprendidas entre los 21 y 70 años de edad y por lo menos tienen cinco años o más de residencia en ese país (2.6 millones), grupos que comparamos con los inmigrantes mexicanos que llegaron con 16 años o más (6.8 millones) ambos grupos clasificados por condición de ciudadanía estadounidense.

En 1982 la Corte Suprema de Estados Unidos bajo la ley Plyler v. Doe garantiza a los menores el derecho a una educación primaria y secundaria ( $k$-12), independientemente de su estatus migratorio. Los inmigrantes que llegaron en la infancia tuvieron una opción para su desarrollo, asistir a la escuela, aun cuando las leyes impuestas desde entonces no contemplan integrarlos plenamente a la sociedad estadounidense. La edad de llegada y la obtención de la ciudadanía favorecen alcanzar mejores niveles de escolaridad y dominio del idioma inglés 
entre los inmigrantes mexicanos, lo que favorece sus oportunidades de integración (Cuadro 3 y 4).

La proporción de llegados en la infancia con y sin ciudadanía con baja escolaridad es inferior respecto de los llegados con 16 años o más de edad. La proporción de los llegados en la infancia sin ciudadanía estadounidense $(37.7 \%)$ es inferior respecto de los naturalizados que llegaron con 16 años o más (47.8\%). Entre los que tienen estudios superiores, el 23.8\% de los llegados en la infancia con ciudadanía tienen escolaridad alta, proporción que es superior en 10.9 puntos porcentuales por arriba de los inmigrantes mexicanos que llegaron con 16 años o más naturalizados (12.8\%). Destaca, la mayor proporción de llegados en la infancia sin ciudadanía estadounidense con estudios superiores respecto de los llegados con 16 años o más, 9.1\% contra 5.5\% respectivamente.

Para la interpretación del índice de disimilitud (ID) el grupo de referencia son los llegados en la infancia con ciudadanía estadounidense. El 21.7\% de los llegados en la infancia sin ciudadanía estadounidense tendría que cambiar su nivel de escolaridad para tener una distribución similar al grupo de referencia, esa relación entre que llegaron con 16 años o más con y sin ciudadanía estadounidense es de $27.5 \%$ y $42.0 \%$ respectivamente. Resultado que muestra que no obstante que los llegados en la infancia no naturalizados tienen desventajas por su estatus de residencia alcanzan niveles de escolaridad similares a los se han naturalizado $y$, se alejan del perfil tradicional de baja calificación de los que llegan en edad laboral (Cuadro 3). Los resultados del ID permiten corroborar que a menor edad al ingreso al país de acogida los inmigrantes mexicanos logran mejores niveles de escolaridad, factor que se relaciona de manera positiva con la obtención de la ciudadanía estadounidense. Si bien, la edad de llegada favorece niveles más altos de escolaridad, la ciudadanía es un mejor predictor de los resultados educativos entre los inmigrantes mexicanos, pues la ciudadanía les amplía sus derechos y acceso a beneficios sociales y económicos.

Con todas las barreras impuesta para su desarrollo, los llegados en la infancia se esfuerzan por mejorar su situación aprovechando las limitadas oportunidades que se abren para su desarrollo, motivados por las mismas razones por las que un día sus padres decidieron llevarlos a Estados Unidos, mejorar su situación económica y condiciones de vida. Se han identificado para los estudiantes en situación irregular de residencia como barreras para el acceso a la educación superior las distintas leyes que regulan la educación superior a nivel estatal. Muchos de ellos asumen de antemano que no pueden asistir debido a la información limitada que proporcionan las escuelas de postsecundaria, falta de financiamiento para cubrir la matrícula y costos de vida, la necesidad de trabajar, altos índices de pobreza y el temor de compartir información sobre su situación legal y la de sus familiares (Jaimes Pérez, 2014). 
Cuadro 3 - Índice de disimilitud y nivel de escolaridad. Inmigrantes mexicanos en Estados Unidos de 21 a 70 años de edad por edad de llegada y condición de ciudadanía estadounidense, 2017

\begin{tabular}{l|c|c|c|c}
\hline & \multicolumn{2}{|c|}{ Llegaron en la infancia } & Llegaron con 16 años o más de edad \\
\hline $\begin{array}{c}\text { Nivel de } \\
\text { escolaridad }\end{array}$ & Con ciudadanía & Sin ciudadanía & Con ciudadanía & Sin ciudadanía \\
\hline Total & 100.0 & 100.0 & 100.0 & 100.0 \\
\hline Baja/1 & 22.6 & 37.7 & 47.8 & 63.6 \\
\hline Media/2 & 53.6 & 53.2 & 39.3 & 30.9 \\
\hline Alta/3 & 23.8 & 9.1 & 12.8 & 5.5 \\
\hline $\begin{array}{l}\text { ID del nivel de } \\
\text { escolaridad }\end{array}$ & 21.7 & 27.5 & 42.0 \\
\hline $\begin{array}{l}1 / \text { Hasta el 12o grado sin diploma. 2 / Diploma de escuela secundaria, 1 o más años de grado } \\
\text { universitario, sin título, 3 / Estudios superiores. }\end{array}$ \\
\hline
\end{tabular}

Fuente: Estimación propia con base en U.S. Census Bureau, American Community Survey (ACS), 2017.

Un segundo factor relevante para la integración de los inmigrantes en la sociedad norteamericana, en particular en los diferentes mercados laborales es el dominio del idioma inglés (Santibáñez, Lavore, 2012). La irregularidad de residencia y la barrera lingüística afecta prácticamente todos los ámbitos de la vida de los inmigrantes, el económico, social y cultural. Nuestros resultados confirman los hallazgos de Bleakley y Chin (2004), los inmigrantes que ingresaron a Estados Unidos siendo niños tienen mejor conocimiento del idioma inglés, 91.5\% de los llegados en la infancia naturalizados hablan muy bien y bien el idioma inglés, relación que es del $76.3 \%$ entre los que no cuentan con la ciudadanía estadounidense, cifra que es superior al de los inmigrantes mexicanos que llegaron con 16 años o más de edad naturalizados (67.2\%). Nuevamente ID muestra una gran similitud entre los llegados en la infancia con y sin ciudadanía estadounidenses y los logros de los llegados en la infancia sin ciudadanía, entre los que se encuentran los indocumentados, tan solo el $17.4 \%$ tendría que mejorar su dominio del idioma inglés para alcanzar la misma distribución de los naturalizados. Brecha que marca una importante diferencia con respecto al grupo de mexicanos que Ilegaron a Estados Unidos con 16 años o más de edad con y sin ciudadanía estadounidense, los cuales son marcadamente disimiles en cuando a domino del idioma inglés se refiere. La edad de llegada y la obtención de la ciudadanía cambian el perfil tradicional de baja calificación de los inmigrantes mexicanos, pues los llegados en la infancia tiene mayores logros educativo y dominio del idioma inglés (cuadro 3 y 4).

Es común utilizar el dominio del idioma inglés como indicador para medir la integración cultural, pero éste es cada vez más un requisito de los mercados globales, por lo que también es un indicador de capital humano, dominar dos o más idiomas lejos de ser una desventaja se asocia con múltiples beneficios en el 
mercado laboral (Gaspar, 2018). Sin embargo, cuando los niños o sus padres o el conjunto de la familia carecen de dominio del inglés pueden estar en desventajas (Child Trend DataBanck, 2014). Una alternativa para medir la integración cultural es el indicador de hogares lingüísticamente aislados (Gaspar, 2016). Nueve de cada diez mexicanos que llegaron en la infancia y se han naturalizado viven en hogares donde solo se habla inglés, esa relación entre los que no se han naturalizado es de ocho de cada diez. "Tales resultados muestran que los inmigrantes mexicanos que llegaron en la infancia superan obstáculos y se adaptan, pero el contexto social y de desarrollo tienen considerable importancia en los procesos de adaptación y movilidad social (Rumbaut, 2006)" (Gaspar, 2018, p. 190). Los datos ID nuevamente confirman la mayor similitud entre inmigrantes mexicanos que llegaron en la infancia al observar indicadores más alto de dominio del idioma inglés respecto de los que llegaron con 16 años o más de edad, la disimilitud es alta aun entre los que cuentan con ciudadanía estadounidense al alcanzar un ID de 40.1\% (Cuadro 4).

Cuadro 4 - Índice de disimilitud (ID) y dominio del idioma inglés. Inmigrantes mexicanos en Estados Unidos de 21 a 70 años de edad por edad de llegada y condición de ciudadanía estadounidense, 2017

\begin{tabular}{l|c|c|c|c}
\hline \multicolumn{1}{c|}{ Hablan Inglés } & \multicolumn{2}{|c|}{ Llegados en la infancia } & \multicolumn{2}{c}{ Llegados con 16 años o más } \\
\hline Total & $\begin{array}{c}\text { Con } \\
\text { ciudadanía }\end{array}$ & $\begin{array}{c}\text { Sin } \\
\text { ciudadanía }\end{array}$ & $\begin{array}{c}\text { Con } \\
\text { ciudadanía }\end{array}$ & $\begin{array}{c}\text { Sin } \\
\text { ciudadanía }\end{array}$ \\
\hline Muy bien y bien & 100.0 & 100.0 & 100.0 & 100.0 \\
\hline No bien y no hablan inglés & 91.5 & 76.3 & 67.2 & 39.6 \\
\hline Índice de disimilitud (ID*100) & 8.5 & 23.7 & 32.8 & 60.4 \\
\hline $\begin{array}{l}\text { \% en hogares lingüísticamente no } \\
\text { aislado }\end{array}$ & c.r. & 17.4 & 40.1 & 58.4 \\
\hline
\end{tabular}

Cf: Categoría de referencia. Llegados en la infancia con ciudadanía estadounidense.

Fuente: Estimación propia con base en U.S. Census Bureau, American Community Survey (ACS), 2017.

Participar en el mercado laboral "es la base de la estructura social, por eso los cambios en este nivel reflejan y repercuten directamente en la estructura de la sociedad en clases, estratos y grupos sociales" (Canales, 2002, p. 20). Si bien la integración laboral no es el único factor de referencia para una integración exitosa, sí resulta ser una medida fundamental de equidad y oportunidades que refleja el éxito de los inmigrantes en la sociedad estadounidense (Gozdziak, Martin, 2005). "Incorporarse al mercado laboral es una de las primeras necesidades que los inmigrantes deben cubrir, de ello depende su permanencia en el país vecino, más aún si se trata de una población que se caracteriza por su perfil predominantemente laboral, como es el caso de la inmigración mexicana" (Gaspar, 2018, p. 192).

De acuerdo con la estadística (Cuadros 5-7). 
1) los inmigrantes mexicanos que llegaron en la infancia con y sin ciudadanía estadounidense tienen TPE mayor a las observadas por los inmigrantes que llegaron con 16 años o más de edad.

2) Sin distinción del grupo, más de ocho de cada diez logra emplearse todo el año y en proporciones similares trabajan a tiempo completo. Resultado indicativo de la importancia de los mexicanos para el mercado laboral estadounidense.

3) La mayoría de los inmigrantes mexicanos trabajan en empresas privadas, pero los llegados en la infancia naturalizados se emplean en mayor proporción en el gobierno (15.2\%), al igual que los llegados con a 16 años o más de edad con ciudadanía estadounidense (8.7\%). Es probable que este grupo goce de mejores condiciones contractuales como resultado de su condición de ciudadanía.

4) El autoempleo es más alto entre los no naturalizado, pero es probable que el auto empleo sea más estables entre los naturalizados.

Cuadro 5 - Indicadores laborales. Inmigrantes mexicanos en Estados Unidos de 21 a 70 años de edad por edad de llegada y condición de ciudadanía estadounidense, 2017

\begin{tabular}{l|c|c|c|c}
\hline \multirow{2}{*}{} & \multicolumn{2}{|c|}{ Llegados en la infancia } & \multicolumn{2}{c}{ Llegados con 16 años o más } \\
\cline { 2 - 5 } & $\begin{array}{c}\text { Con } \\
\text { ciudadanía }\end{array}$ & $\begin{array}{c}\text { Sin } \\
\text { ciudadanía }\end{array}$ & $\begin{array}{c}\text { Con } \\
\text { ciudadanía }\end{array}$ & $\begin{array}{c}\text { Sin } \\
\text { ciudadanía }\end{array}$ \\
\hline Tasa de participación económica (TPE) & 76.0 & 72.9 & 69.2 & 68.7 \\
\hline Tasa de ocupación (TO) & 96.2 & 95.0 & 96.7 & 95.8 \\
\hline \% se empleó todo el año & 86.5 & 85.1 & 86.9 & 84.4 \\
\hline \% trabajó a tiempo completo & 84.6 & 82.9 & 85.8 & 84.6 \\
\hline Empleado de: & 100.0 & 100.0 & 100.0 & 100.0 \\
\hline \multicolumn{1}{|c|}{ Empresa privada } & 77.5 & 87.1 & 78.7 & 84.3 \\
\hline Gobierno & 15.2 & 4.2 & 8.7 & 2.3 \\
\hline Autoempleo & 7.2 & 8.5 & 12.4 & 13.1 \\
\hline Autoempleo-informal & 5.0 & 6.9 & 8.8 & 10.9 \\
\hline Autoempleo-formal & 2.2 & 1.6 & 3.6 & 2.2 \\
\hline
\end{tabular}

Fuente: Estimación propia con base en U.S. Census Bureau, American Community Survey (ACS), 2017.

5) Los llegados en la infancia naturalizados logran una mejor posición en la escala ocupacional, $32.0 \%$ se inscribe en actividades profesionales. Aun entre los llegados en la infancia no naturalizados el porcentaje que logra ocuparse en actividades profesionales es 3.1 puntos porcentuales por debajo de los que llegaron con 16 años o más de edad naturalizados. Este resultado permite suponer que la edad temprana de llegada favorece no solo el capital humano de los inmigrantes también su incorporación laboral. 
6) El ID corrobora una mejor posición ocupacional para los llegados en la infancia naturalizado y la mayor similitud ocupacional entre los llegados en la infancia no naturalizados y entre los llegados con 16 años o más de edad naturalizados.

Cuadro 6 - Índice de disimilitud y tipo de ocupación. Inmigrantes mexicanos en Estados Unidos de 21 a 70 años de edad por edad de llegada y condición de ciudadanía estadounidense, 2017

\begin{tabular}{|c|c|c|c|c|}
\hline \multirow[b]{2}{*}{ Tipo de ocupación } & \multicolumn{2}{|c|}{ Llegados en la infancia } & \multicolumn{2}{|c|}{ Llegados con 16 años o más } \\
\hline & $\begin{array}{l}\text { Con } \\
\text { ciudadanía }\end{array}$ & $\begin{array}{l}\text { Sin } \\
\text { ciudadanía }\end{array}$ & $\begin{array}{c}\text { Con } \\
\text { ciudadanía }\end{array}$ & $\begin{array}{l}\text { Sin } \\
\text { ciudadanía }\end{array}$ \\
\hline Total & 100.0 & 100.0 & 100.0 & 100.0 \\
\hline Profesionales y relacionadas & 32.0 & 15.4 & 18.5 & 7.1 \\
\hline Servicios, ventas, administración & 25.3 & 20.8 & 17.6 & 10.7 \\
\hline $\begin{array}{l}\text { Limpieza de edificios y } \\
\text { mantenimiento/1 }\end{array}$ & 12.0 & 20.2 & 20.7 & 29.3 \\
\hline Cultivo, pesca y silvicultura & 1.6 & 4.3 & 2.9 & 7.3 \\
\hline $\begin{array}{l}\text { Construcción, mantenimiento y } \\
\text { reparación }\end{array}$ & 12.5 & 21.0 & 16.2 & 24.7 \\
\hline Transporte y producción & 16.3 & 18.0 & 23.9 & 20.7 \\
\hline Extracción & & & & 0.2 \\
\hline Índice de disimilitud ID (\%) & c.r. & 27.9 & 27.8 & 45.1 \\
\hline
\end{tabular}

1/Incluye: Preparación de alimentos.

cf: Categoría de referencia. Llegados en la infancia con ciudadanía estadounidense.

Fuente: Estimación propia con base en U.S. Census Bureau, American Community Survey (ACS), 2017.

La importancia de los inmigrantes mexicanos en la economía y la sociedad estadounidenses está marcada particularmente por situaciones que giran en torno a la percepción y valoración social que se les da, y en menor medida a su tiempo de estancia cuando se compara con su mismo grupo de origen, en otro caso también a su estatus legal de residencia (García, Gaspar, 2019). Si bien los datos muestran que haber llegado antes de los 16 años mejora no sólo las oportunidades educativas, obtención de la ciudadanía estadounidense, dominio del idioma inglés, mejor posición en la escala ocupacional, cuando observamos los niveles de ingreso salarial este efecto positivo sólo se percibe entre los que se naturalizan. Lo que hace suponer que el acceso a la ciudadanía es un recurso para mejorar sus salarios y reducir la desigualdad. La obtención de la ciudadanía, les da protección legal a los inmigrantes, promueve la inversión y permite el acceso a una gama amplia de puestos de trabajo, mejora los salarios, y las contribuciones al fisco, e incrementan el consumo de bienes y servicios (Lynch, Oakford, 2013; Enchautegui, Giannarelli, 2015; Johnson, 2015). 
7) Como se esperaría los llegados en la infancia y los llegados con 16 años o más naturalizados exhiben mejores ingresos que sus contrapartes que no se han naturalizado. Los llegados en la infancia naturalizado ganan en promedio $27.4 \%$ más que los que no se han naturalizado. En todas las ocupaciones los inmigrantes mexicanos que no se han naturalizado ganan menos que su contraparte naturalizada (Cuadro 7).

Cuadro 7 - Salario promedio anual por tipo de ocupación. Inmigrantes mexicanos en Estados Unidos de 21 a 70 años de edad por edad de llegada y condición de ciudadanía estadounidense, 2017

\begin{tabular}{l|c|c|c|c|c|c}
\hline & \multicolumn{3}{|c|}{ Llegados en la infancia } & \multicolumn{2}{c}{ Llegados con 16 años o más } \\
\hline & $\begin{array}{c}\text { Con } \\
\text { ciudadanía }\end{array}$ & $\begin{array}{c}\text { Sin } \\
\text { ciudadanía }\end{array}$ & $\begin{array}{c}\text { Diferencia } \\
\text { porcentual }\end{array}$ & $\begin{array}{c}\text { Con } \\
\text { ciudadanía }\end{array}$ & $\begin{array}{c}\text { Sin } \\
\text { ciudadanía }\end{array}$ & $\begin{array}{c}\text { Diferencia } \\
\text { porcentual }\end{array}$ \\
\hline $\begin{array}{l}\text { Profesionales y } \\
\text { relacionadas }\end{array}$ & $54,150^{\mathrm{a}}$ & $39,323^{\mathrm{b}}$ & 27.4 & $59,955^{\mathrm{c}}$ & $48,633^{\mathrm{d}}$ & 18.9 \\
\hline $\begin{array}{l}\text { Servicios, ventas, } \\
\text { administración }\end{array}$ & $33,482^{\mathrm{a}}$ & $25,481^{\mathrm{b}}$ & 23.9 & $34,199^{\mathrm{c}}$ & $27,927^{\mathrm{d}}$ & 18.3 \\
\hline $\begin{array}{l}\text { Limpieza de } \\
\text { edificios y } \\
\text { mantenimiento/1 }\end{array}$ & $25,526^{\mathrm{a}}$ & $21,578^{\mathrm{b}}$ & 15.5 & $26,916^{\mathrm{c}}$ & $22,805^{\mathrm{d}}$ & 15.3 \\
\hline $\begin{array}{l}\text { Cultivo, pesca y } \\
\text { silvicultura }\end{array}$ & $22,435^{\mathrm{a}}$ & $21,732^{\mathrm{b}}$ & 3.1 & $26,389^{\mathrm{c}}$ & $22,892^{\mathrm{d}}$ & 13.3 \\
\hline $\begin{array}{l}\text { Construcción, } \\
\text { mantenimiento y } \\
\text { reparación }\end{array}$ & $44,012^{\mathrm{a}}$ & $34,148^{\mathrm{b}}$ & 22.4 & $43,904^{\mathrm{c}}$ & $34,584^{\mathrm{d}}$ & 21.2 \\
\hline $\begin{array}{l}\text { Transporte y } \\
\text { producción }\end{array}$ & $38,603^{\mathrm{a}}$ & $31,320^{\mathrm{b}}$ & 18.9 & $37,487^{\mathrm{c}}$ & $29,467^{\mathrm{d}}$ & 21.4 \\
\hline
\end{tabular}

Prueba Z para comparar proporciones de columna. Cada letra del subíndice denota un subconjunto de categoría cuyas proporciones no difieren de forma significativa entre sí en el nivel 0.05 .

1/Incluye: Preparación de alimentos.

Fuente: Estimación propia con base en U.S. Census Bureau, American Community Survey (ACS), 2017.

Crear un camino hacia la ciudadanía y permitirles trabajar legalmente a los más de 11 millones de indocumentados aumentaría sus salarios y contribuciones estatales y locales en más de \$2.1 mil millones al año (ITEP, 2016). La naturalización como señala Gilbertson y Singer (2003 citado en Enchautegui y Giannarelli, 2015) puede ser una estrategia trasnacional para mantener vínculos en más de un país. Para Enchautegui y Giannarelli los inmigrantes abordan la cuestión de la ciudadanía no solo a nivel individual también a través de la experiencia de su grupo nacional, pero es el Estado el que juega un papel relevante en las decisiones de naturalización y las políticas de integración del país anfitrión, que crean experiencias colectivas que podrían promover o desalentar la naturalización. 


\section{Conclusiones}

Dado el contexto actual de la política migratoria que prevalece en Estados Unidos (deportación y reducción de canales legales de ingreso para los migrantes), este estudio muestra los cambios sociodemográficos y laborales que han experimentado los inmigrantes mexicanos en ese país por edad de llegada y condición de ciudadanía. Se muestra que la obtención de la ciudadanía estadounidense para los llegados en la infancia mejora su capital humano, posición en la escala ocupacional y sus ingresos por trabajo, en consecuencia sus aportaciones monetarias al fisco. Los inmigrantes que llegaron en la infancia a Estados Unidos contribuyen al crecimiento económico de la nación que los ha acogido, aunque con cierta resistencia, durante sus vidas lo que les ha permito nutrir su capital humano, formar una familia y acumulado activos.

Los inmigrantes que llegaron en la infancia no naturalizados o que no han regulado estatus legal de residencia, mejoran su capital humano en cuanto a nivel de escolaridad y dominio del idioma ingles y acumulan activos (42.0\% vive en casa propia o con hipoteca, el $90 \%$ vive en hogares con al menos un vehículo). Sin embargo, observan una inserción laboral desventajosa respecto de los naturalizados y su situación los coloca en riesgo de perder sus activos si son deportados.

De hecho, el índice de disimilitud para los llegados en la infancia con y sin ciudadanía estadounidense, indican que las características de capital humano y ocupacional que exhiben se aleja de las observadas por los inmigrantes mexicanos que llegaron con 16 años o más de edad, particularmente de aquellos que no se han naturalizado o regulado su situación de residencia legal. Es decir, los inmigrantes que llegaron en la infancia con y sin ciudadanía estadounidense se alejan del perfil tradicional de baja calificación que ha caracterizado a la migración laboral procedente de México.

Hill y Wiehe (2018) del ITEP (Institute on Taxation and Economic Policy) encontraron que los 11 millones de inmigrantes indocumentados que viven y trabajan en los Estados Unidos contribuyen con más de $\$ 11,74$ mil millones en impuestos estatales y locales. Los 1.3 millones de jóvenes inmigrantes indocumentados inscritos o elegibles para DACA contribuyen con $\$ 1.7$ millones al año, aproximadamente pagan en promedio $8.3 \%$ de sus ingresos en impuestos. Cabe recordar que los inmigrantes mexicanos que llegaron en la infancia encabezan este grupo $(78.7 \%)$.

La importancia de resaltar los cambios en el perfil de los llegados en la infancia, en particular para los que no se han naturalizado o regulado su situación migratoria, fue para mostrar la importancia del programa DACA como una opción, aunque temporal, que les permite permanecer en Estados Unidos y la relevancia que tienen los requisitos de elegibilidad, como la edad actual, que dejan fuera a un importante número candidatos sin posibilidades de regular su situación migratoria. 
Es común enfocar los esfuerzos de investigación a demostrar que los inmigrantes traen desventajas económicas a la nación, otras en cambio muestran lo contrario, es decir que las ventajas económicas y beneficios superan cualquier desventaja de la inmigración. La mayoría de los estudios se olvidan que no se trata de números sino de personas individuales o en familia, estas últimas conformadas por miembros con diferente estatus de residencia y nacionalidad, $70 \%$ de los destinatarios de DACA tiene un cónyuge, hijo o hermano nacido en Estados Unidos (Hill, Wiehe, 2018).

Entre los llegados en la infancia procedentes de México no naturalizados o en situación irregular de residencia $53.0 \%$ es jefe o cónyuge y 20\% hijo. En más de 1.3 millones de hogares hay al menos un inmigrante mexicano no naturalizado o en situación irregular de residencia que llegó en la infancia. Hogares que albergan a más de 6.5 millones de personas (53.5\% nació en México, 45.0\% en Estados Unidos y $1.6 \%$ en otro país). Estas cifras dan cuenta de la magnitud del impacto que tienen sobre las familias las decisiones que se tomen en torno a los llegados en la infancia no naturalizados o en situación irregular de residencia. Tómese en consideración que este es solo el caso de los procedentes de México, pues el total de afectados es de más de 2.5 millones de hogares y casi 10 millones de personas.

Rescindir el programa y mantener una postura excluyente sobre los llegados en la infancia perjudica no solo a los que han cumplido con los requisitos establecidos para participar en el programa DACA, también a los que han quedado fuera por los requisitos establecidos, los afecta de manera individual y familiar. La evidencia mostrada por numerosos estudios constata que los llegados en la infancia de media y alta calificación impulsan la innovación y el crecimiento económico. También los menos calificados ocupan puestos para los cuales hay una oferta laboral insuficiente, y los llegados en la infancia que han alcanzado la edad de adulto mayor ya sirvieron y contribuyeron a la economía de la nación en la que crecieron.

El activismo social de los indocumentados refleja el fuerte deseo de ser integrados como miembros plenos de la sociedad estadounidense; como se ha constatado a lo largo del estudio, los inmigrantes en situación irregular están trabajando o estudiando y muchos de ellos combinan ambas actividades. El activismo de los migrantes se convierte en un amplio movimiento nacional heterogéneo que reivindica su derecho a legalizar su situación migratoria y acceder sin discriminaciones al mercado laboral y al sistema educativo de Estados Unidos.

Frente a la importancia económica y social de los inmigrantes mexicanos llegados en la infancia a Estados Unidos y el reto de regularización migratoria de una parte significativa de ellos para lograr derechos sociales y políticos plenos, acceder a la educación, la salud y mejores mercados laborales con un mayor aporte al relevo generacional ocupacional, al sistema de pensiones y recaudación fiscal, 
al mismo tiempo mayor ingreso y bienestar familiar, es muy importante reconocer la postura de política adversa contra los migrantes y la reforma migratoria durante el gobierno de Trump.

\section{Referencias bibliográficas}

AMERICAN COMMUNITY SURVEY. 2017. Disponible en: <https://www.census.gov/ programs-surveys/acs $>$.

AMERICAN IMMIGRATION COUNCIL. The Dream Act, DACA, and Other Policies Designed to Protect Dreamers. 2019. Disponible en: <https:/www. americanimmigrationcouncil.org/research/how-united-states-immigration-systemworks?gclid=CjOKCQjwtsv7BRCmARIsANu-CQfDfqFJ_9sB1rUKMbckA4ogn_3SnV 545eGSNiyNeBzwwJCKHbgoWbkaAhc5EALw_wcB>.

AMERICAN IMMIGRATION COUNCIL. The Dream Act, DACA, and Other Policies Designed to Protect Dreamers. 2017. Disponible en: <https://www. americanimmigrationcouncil.org/sites/default/files/research/the_dream_act_daca_ and_other_policies_designed_to_protect_dreamers.pdf $>$.

ASUMI. Case: 18-15068, 03/20/2018, ID: 10805944, DktEntry: 73, Page 1 of 60. Consolidated Case Nos. 18-15068, 18-15069, 18-15070, 18-15071, 18-15072, 18-15128, 18-15133, 18-15134. Disponible en: <http://cdn.ca9.uscourts.gov/ datastore/general/2018/03/28/18-15068-102-companies-amicus-brief.pdf $>$.

BAUM, Sandy; FLORES, Stella M. Higher education and children in immigrant families. The future of children, v. 21, n. 1, p. 171-193, 2011.

BIER, David. Ending DACA Will Impose Billions in Employer Compliance Costs. CATO, Sept. 1, 2017. Disponible en: <https://www.cato.org/blog/ending-daca-willimpose-billions-employer-compliance-costs $>$.

BLEAKLEY, Hoyt; CHIN, Aimeé. Language skills and earnings: Evidence from childhood immigrants. The Review of Economics and Statistics, v. 86, n. 2, p. 481-496, 2004.

BORJAS, George J.; TIENDA, Marta. The employment and wages of legalized immigrants. International Migration Review, v. 27, n. 4, p. 712-747, 1993.

CANALES CERÓN, Alejandro. Migración y trabajo en la era de la globalización: el caso de la migración México-Estados Unidos en la década de 1990. Papeles de Población, v. 8, n. 33, p. 47-80, 2002.

CHILD TREND DATA BANCK. Dual Language Learners. 2004. Disponible en: < https:// www.childtrends.org/wp-content/uploads/2014/11/127_Dual_Language_Learners.pdf $>$.

DUNCAN, Otis Dudley; DUNCAN, Berly. A methodological analysis of segregation indexes. American Sociological Review, v. 20, n. 2, p. 2010, 2017, 1955.

ENCHAUTEGUI, María E.; GIANNARELLI, Linda. The Economic Impact of Naturalization on Immigrants and Cities. Urban Institute. Research report, 2015. Disponible en: <https://www.urban.org/sites/default/files/publication/76241/2000549-The-Econo mic-Impact-of-Naturalization-on-Immigrants-and-Cities.pdf $>$.

ENG, David. I was a dreamer, too-and I know America is stronger for welcoming immigrants like my parents. 21.02.2018. Disponible en: <https://qz.com/1212376/ 
daca-i-was-a-dreamer-too-and-i-know-america-is-stronger-for-welcomingimmigrants-like-my-parents/ $>$.

GARCÍA ZAMORA, Rodolfo; GASPAR OLVERA, Selene. Llegados en la infancia. Todos somos soñadores. In: PIÑEIRO, Rodolfo Cruz; VARGAS VALLE, Eunice D.; HERNÁNDEZ, Alberto; LÓPEZ JARAMILLO, Ana María (coords.). Los Dreamers. Ante un escenario de cambio legislativo: Inserción social y económica en México. Colegio de la Frontera Norte (COLEF), 2019, p. 183-206.

GASPAR OLVERA, Selene. Integración de los inmigrantes mexicanos que llegaron en la infancia a Estados Unidos. In: La situación Demográfica de México 2017. México: Consejo Nacional de Población, CONAPO, 2018, p. 177-202.

GASPAR OLVERA, Selene. ¿Estudiar para emigrar o emigrar para estudiar? Procesos de integración de los inmigrantes mexicanos calificados en Estados Unidos. Tesis de Maestría en Demografía Social, UNAM, Posgrado de la Facultad de Ciencias Políticas y Sociales, 2016.

GLICK, Jennifer E.; WALKER, Laquitta; LUZ, Luciana. Linguistic isolation in the home and community: Protection or risk for young children?. Social science research, v. 42, n. 1, p. 140-154, 2013.

GOZDZIAK, Elzbieta; MARTIN Susan F. (eds.). New Immigrant Communities: Addressing Integration Challenges. Lanham, Maryland: Lexington Books, 2005.

HERMANSEN, Are Skeie. Immigrant Children's. Age at Arrival, Skill Formation, and Adult Socioeconomic Success. Oslo: University of Oslo, 2013.

HILL, Misha E.; WIEHE Meg. State \& Local Tax Contributions of Young Undocumented Immigrants. Institute on Taxation \& Economic Policy. 2018. Disponible en: < https:// itep.org/wp-content/uploads/2018DACA.pdf $>$.

ITEP. The Institute on Taxation and Economic Policy. Undocumented Immigrants' State \& Local Tax Contributions. 2016. Disponible en: < https://itep.sfo2.digitaloceanspaces. com/immigration2016-1492632815.pdf >.

JAIMES PÉREZ, Zenen. Removing Barriers to Higher Education for Undocumented Students. Center for American Progress, 2014. Disponible en: <https://www. luminafoundation.org/files/resources/removing-barriers-for-undocumented-students.pdf $>$.

JOHNSON, Randel K. Restructuring the U.S. immigration system to increase security and promote economic growth. U.S. Chamber of Commerce, 2015. Disponible en: $<$ https://www.uschamber.com/sites/default/files/uscc_restructuring_u.s._imm_62015.pdf $>$.

KROGSTAD, Jeans Manuel. Americans broadly support legal status for immigrants brought to the U.S. illegally as children. Pew Research Center, 2020. Disponible en: <https://www.pewresearch.org/fact-tank/2020/06/17/americans-broadly-supportlegal-status-for-immigrants-brought-to-the-u-s-illegally-as-children/> .

LAWLOGIX. The Initial DREAM Act S.1291 (2001). 2013. Disponible en: <https:// www.lawlogix.com/what-is-the-dream-act-and-who-are-dreamers/>.

LEE, Youngro. To Dream or Not to Dream: A Cost-Benefit Analysis of the Development, Relief, and Education for Alien Minors (Dream) Act. Cornell Journal of Law and Public Policy, v. 16, n. 1, Article 5, 2006. Disponible en: <http://scholarship.law. cornell.edu/cjlpp/vol16/iss1/5>. 
LYNCH, Robert; OAKFORD, Patrick. The Economic Effects of Granting Legal Status and Citizenship to Undocumented Immigrants. Center for American Progress, 2013. Disponible en: <https://www.americanprogress.org/issues/immigration/ reports/2013/03/20/57351/the-economic-effects-of-granting-legal-status-andcitizenship-to-undocumented-immigrants/ $>$.

PASTOR, Manuel; SCOGGINS, Justin. Citizen Gain: The Economic Benefits of Naturalization for Immigrants and the Economy. Center for Study of Immigrant Integration CSII, 2012. Disponible en: <https://dornsife.usc.edu/assets/sites/731/ docs/citizen_gain_web.pdf $>$.

PORTES, Alejandro; RUMBAUT, Rubén G. América inmigrante. Barcelona: Anthropos, 2010.

RUMBAUT, Rubén G.; EWING, Walter. The Myth of Immigrant Criminality and the Paradox of Assimilation. American Immigration Council, Special Report. 2007. Disponible en: < https://www.americanimmigrationcouncil.org/research/myth-imm igrant-criminality-and-paradox-assimilation $>$.

RUMBAUT, Rubén G. Edades, etapas de la vida y cohortes generacionales: un análisis de las dos primeras generaciones de inmigrantes en Estados Unidos. In: PORTES, Alejandro; DeWIND, Josh (coords.). Repensando las migraciones. Nuevas perspectivas teóricas y empíricas. Zacatecas: Universidad Autónoma de Zacatecas, Miguel Ángel Porrúa, Red UAZ, 2006, p. 361-410.

SANTIBAÑEZ ROMELLÓN, Jorge; LAVORE FANJUL, Elisa. Los jóvenes inmigrantes mexicanos en Estados Unidos. In: RAMÍREZ GARCÍA, Telésforo; CASTILLO, Manuel Ángel (coords.). México ante los recientes desafíos de la migración internacional. México: CONAPO, 2012, p. 51-86. Disponible en: < http://imumi.org/attachments/ mexico_recientes_desafios.pdf $>$.

USCIS. Number of Form I-821D, Consideration of Deferred Action for Childhood. Arrivals, by Fiscal Year, Quarter, Intake and Case Status Fiscal Year 2012-2020. 15.08.2012-03.03.2020. Disponible en: <https://www.uscis.gov/sites/default/files/ document/data/DACA_performancedata_fy2020_qtr2.pdf $>$.

YATES, Laura S. Plyler v. Doe and the Rights of Undocumented Immigrants to Higher Education: Should Undocumented Students Be Eligible for In-State College Tuition Rates?. Washington University Law Review, v. 82, n. 2, 2004. Disponible en: < https:// openscholarship.wustl.edu/cgi/viewcontent.cgi ?article $=1375 \&$ context $=$ law lawreview $>$.

WATERS Mary C.; GERSTEIN PINEAU, Marisa (eds.). The Integration of Immigrants into American Society. Washington: National Academies of Science, Engineering, and Medicine, 2015. Disponible en: <https://www.nap.edu/catalog/21746/theintegration-of-immigrants-into-american-society $>$.

WOODROW-LAFIELD, Karen A.; XU, Xiaohe; KERSEN, Thomas; POCH, Bunnak. Naturalización de inmigrantes de los estados unidos: destacados de diez países. Investigación de la población y revisión de políticas, v. 23, n. 3, p. 187-218, 2004. Disponible en: <https://www.ncbi.nlm.nih.gov/pmc/articles/PMC2613363/\#R36>. 